reviewing arthropathies, multiple sclerosis, diabetes mellitus, coeliac disease, liver disease, acute leukaemia and Hodgkin's disease, and finally trophoblastic tumours. Unfortunately, the genetic linkage between HLA and 21-hydroxylase deficiency was discovered too late for inclusion in the Bulletin. 21-hydroxylase deficiency is a paradigm of genetic disease as the genetics, biochemistry, and treatment are so well worked out. Never before have we had such razor-sharp dissection of a segment of a human chromosome and been able to discern, even if we do not always fully understand, the interactions of closely linked genes, the effects of linkage disequilibrium, and the emergence of disease associations. Without doubt, HLA will be of the greatest practical importance in the prediction of those at risk of developing many diseases with a clear genetic basis but which depend on environmental triggers. As our understanding of this system increases, we will be able to detect and potentially remove offending environmental agents thus allowing true prevention.

This issue of the British Medical Bulletin is first rate and is obligatory reading for every clinical geneticist. It is remarkable value at $£ 5.00$ and is perfectly complementary to 'Basic Immunogenetics' by Fudenberg and his colleagues which is reviewed below.

\section{R. HARRIS}

\section{Basic Immunogenetics}

2nd Edition. By H. H. Fudenberg, J. R. L. Pink, An-Chuan Wang, and S. D. Douglas.

(Pp. ix +262 ; Figures + Tables. £3.95.)

New York and Oxford: Oxford University Press. 1978.

Since the first edition was published in 1972, a great deal has happened in the field of immunogenetics and it is remarkable that Dr Fudenberg and his colleagues have managed to limit the expansion of the second edition to only $\mathbf{4 8}$ pages. The use of finer quality paper has also kept the overall dimensions of the book about the same. One has to confess at the outset that immunogenetics is difficult. I wonder, for example, how many immunochemists feel at home in the new and rapidly expanding sister-field of cell immunology? Taken together, the subject matter of this book, which covers the chemistry and genetics of antibody molecules, cell mediated immunology, and human blood group serology, is a highly concentrated collection of facts and theories. One should not, however, succumb to the temptation of leaving immunogenetics to the experts in the hope that it will become easier as time goes by, for already the subject has produced a number of unexpected observations with wide relevance to genetics as a whole, as a few examples will show. Single immunoglobulin polypeptides are coded for by two or more genes; families of multiple closely linked genes are characteristic of immunology, but may well be a basic phenomenon of mammalian germ lines; immunoglobulins demonstrate the importance of somatic mutations; the interaction between different lymphocyte types exerts control over protein synthesis, presumably by some form of genetic feedback. Last, but not least, allelic exclusion (involving autosomes) was discovered in a study of antibody molecules.

Fudenberg's book covers all these areas with great clarity, tackling with skill a plethora of experimental data from both comparative and human studies. An abundance of tables and diagrams is a particularly praiseworthy feature of the book. As in the first edition, chapter 1 is an introductory essay which is well worth reading. Chapter 2 describes immunoglobulin structure and evolution and goes on to a lucid exposition of the admittedly complicated genetics of immunoglobulin molecules. Chapter 4 tackles the generation of antibody variability and describes what is known of the genetics of antibody specificity. Chapter 5 deals succinctly with lymphoid membrane antigens including their genetic control and relation to immune response, $T$ and $B$ cells, and lymphocyte interactions. The potted version of mouse $\mathrm{H}-2$ is excellent. Chapter 6 is an adequate review of the human blood group systems and the book finishes, as before, with a number of useful appendices.

The index is generally good, though there are occasional omissions. For example, I encountered ' $F d$ ' in the text but could not find an entry in the index or in the appendices. This edition went to press before the Seventh International Histocompatibility Workshop and before most of the new information on HLA-DW and -DRW loci became available. (This gap is filled admirably by 'The HLA System' in No. 3 of Vol. 34 of the British Medical Bulletin which should be read in parallel with this book and is reviewed above.) The section on HLA will no doubt be extended in a third edition. These criticisms do not detract significantly from 'Basic Immunogenetics' which remains an essential for the libary of geneticists of all persuasions, both graduate and undergraduate.

R. HARRIS

The Genetics of Aging

Edited by E. L. Schneider. (Pp. xvi +424 ; Figures + Tables. $\$ 39 \cdot 00$.) New York and London: Plenum Press. 1978.

Homer likened the generation of man to the fall of leaves. Addison introduced a continuous stochastic 